\title{
Rire et littérature, Mélanges en l'honneur de Jean Serroy, textes ressemblés par Bernard Roukhomovsky
}

\section{Gabriella Bosco}

\section{(2) OpenEdition Journals}

\section{Edizione digitale}

URL: https://journals.openedition.org/studifrancesi/26983

DOI: $10.4000 /$ studifrancesi.26983

ISSN: 2421-5856

\section{Editore}

Rosenberg \& Sellier

\section{Edizione cartacea}

Data di pubblicazione: 1 avril 2007

Paginazione: 228-229

ISSN: 0039-2944

\section{Notizia bibliografica digitale}

Gabriella Bosco, «Rire et littérature, Mélanges en l'honneur de Jean Serroy, textes ressemblés par Bernard Roukhomovsky», Studi Francesi [Online], 151 (LI | I) | 2007, online dal 30 novembre 2015, consultato il 23 novembre 2021. URL: http://journals.openedition.org/studifrancesi/26983 ; DOI: https://doi.org/10.4000/studifrancesi.26983

Questo documento è stato generato automaticamente il 23 novembre 2021.

\section{cc) (†) $\odot$}

Studi Francesi è distribuita con Licenza Creative Commons Attribuzione - Non commerciale - Non opere derivate 4.0 Internazionale. 


\title{
Rire et littérature, Mélanges en l'honneur de Jean Serroy, textes ressemblés par Bernard Roukhomovsky
}

\author{
Gabriella Bosco
}

\section{NOTIZIA}

Aa. Vv., Rire et littérature, Mélanges en l'honneur de Jean Serroy, textes ressemblés par Bernard RочкномоvsкY, «Recherches \& Travaux», n. 67, 2005, pp. 295.

1 Studi dedicati a Jean Serroy - il suo importante saggio sul Page disgracié terminava sulla considerazione che niente è più serio del ridere - il presente numero di «Recherches \& Travaux» (rivista dell'università Stendhal di Grenoble 3 a lungo diretta dallo stesso Serroy) rende omaggio a quello che, tra i tanti filoni di ricerca perseguiti dal grande secentista, è stato il più costante: il comico in letteratura.

Diviso in tre sezioni, il volume affronta il tema dal punto di vista teorico e nelle sue più varie realizzazioni attraverso i secoli e i generi. Il primo gruppo di saggi, raccolti sotto il titolo $\mathrm{Du}$ rire, $d u$ ridicule et $d u$ comique, analizza la poetica del ridere dal Cinquecento all'Ottocento utilizzando testi di Louis Garon (Stéphane MACÉ), Cureau de la Chambre (Bernard RouкHомоvsкY), Sorel (Jean-Yves ViALLEToN), Rousseau (Jean-François PERRIN) e Du Roure (Daniel BiLous). La seconda sezione, "Rire et récit", analizza il comico narrativo passando dalla prima scena del Roman comique di Scarron (Françoise LÉTOUBLON) al Quart Livre di Rabelais (Christiane DELoINCE-LOUETTE), dal riso in Crébillon (Jean OUDART) alla narrativa di viaggio in epoca romantica (Alain GUYOT), dalle varie modalità di riso nell'opera di Hugo (René BOURGEOIS) alla lettura fin de siècle, tramite Catulle Mendès, dei contes di Perrault in chiave comica (Bertrand VIBERT) al Gaspard des Montagnes di Henri Pourrat (Jacques CHOCHEYRAs), dal riso angoscioso in Dirty di Georges 
Bataille (Jean-François LOUETTE) al riso contemporaneo in Sauvez la reine del grenoblese Jean-Pierre Chanod (Jacqueline BERNARD-BILLIEZ), per finire su un breve curioso inedito intitolato Masque noir et loup blanc, strizzata d'occhi a Jean Serroy (di Claudette ORIOLBOYER).

3 L'ultima parte del volume, Rire à la scène, rire à l'écran, si occupa di comicità teatrale $\mathrm{e}$ cinematografica. Nel primo settore, si passa dai settecenteschi, anonimi, Plaisirs de la tronche (Jean SGARD) alla commedia-balletto La Mandragore di Karol Szymanowski, grottesco anni Venti tratto dal Bourgeois Gentilhomme (Didier VAN MOERE); dai paradossi del riso secondo Dario Fo (Anick BRILLANT-ANNEQUIN) al nuovo spazio comico nel teatro contemporaneo (Mireille LOSCO-LENA) sino al caso specifico di un drammaturgo teoricamente molto lontano dal comico, Michel Vinaver (Bernadette BOST-ROBERT). Quanto al cinema, due sono i contributi, che analizzano il "riso della ragione" nel Dittatore di Charlie Chaplin (François GENTON) e la rappresentazione del corpo - "à se tordre de rire" - nei film comici americani e francesi degli ultimi decenni (Lauretta CLOUGH e Caroline EADES). 